\title{
Isothermal crystallization kinetics of poly(ethylene terephthalate) and poly(methyl methacrylate) blends
}

\author{
A. Al-Mulla* \\ Chemical Engineering Department, Kuwait University, P.O. Box 5969, 13060 Safat, Kuwait
}

Received 25 March 2007; accepted in revised form 4 May 2007

\begin{abstract}
Different kinetic models like the Avrami, Tobin and Urbanovici-Segal models have been applied for determining the isothermal crystallization kinetics of virgin poly(ethylene terephthalate) (PET) and PET/poly(methyl methacrylate) (PMMA) blends. The different compositions investigated were PET90/PMMA10, PET75/PMMA25 and PET50/PMMA50 [wt/wt\%]. The experimental data was fitted using Solver, a non-linear multi-variable regression program and linearization method. The effect of composition variation of PET/PMMA on parameters like crystallization rate constant and crystallization exponent were investigated. Urbanovici-Segal and Avrami models gave the best fit to the experimental data. Tobin model does not seem to fit the experimental data for the systems under investigation. Experimental results indicated that the crystallization rate constant values increased with decreasing temperatures.
\end{abstract}

Keywords: polymer blends and alloys, thermal properties, crystallization kinetics, poly(ethylene terephthalate) (PET), poly(methyl methacrylate) (PMMA)

\section{Introduction}

The commercial significance of semi-crystalline PET and amorphous PMMA is well known and confirmed by a large number of producers throughout the world. PMMA is a rigid commodity thermoplastic, while PET is a ductile engineering polymer. The melt rheological properties of PET and impact strength of PMMA can be improved by blending PET and PMMA. Semi-crystalline polymers like PET exhibit both crystalline and amorphous phases. Blending of an amorphous polymer like PMMA with PET can affect its physical and thermal properties. The balance between the crystalline and amorphous components of both polymers in the new blend is a function of the composition and chemistry of both the polymers as well as the processing conditions like cooling rates and thermal histories, used to generate the end product. Regarding the chemistry of the polymers

*Corresponding author, e-mail: adamalmulla@kuc01.kuniv.edu.kw (C) BME-PT and GTE used to form the blend various factors like, polymer chain structure, and average molecular weight can affect the rate of crystallization of the polymer, when cooled from the melt. PET has an ester repeat unit in the backbone while PMMA has the ester repeat unit in the side chain. Therefore, there is no affinity between PET and PMMA because of incompatibility; hence, an exploration of the crystalline state of PET in presence of PMMA is worth studying.

Bishara et al. [1] has reported the nonisothermal crystallization kinetics of PET/PMMA blends. In this study the crystallization enthalpy $(\Delta H)$ values were found to be dependent on the cooling rate and composition of the blend. Thermogravimetric analysis of PET/PMMA blends were carried out by Al-Mulla et al. [2]. TGA analysis revealed that the blends were heterogeneous in nature. Morphological studies of PET/PMMA blends were also reported in the same work. The blends were found 
to exhibit a heterogeneous morphology. PMMA was found to form spherical particles and were seen dispersed in the PET matrix. Dewangan et al. [3] developed compatibilized PET/PMMA blends using amphiphilic block copolymers of poly(tertbutyl acrylate) and PMMA. The mechanical and rheological properties of the compatibilized blends have been reported.

The physical and mechanical properties of PET/PMMA blends depend on the blend composition, the physical and chemical interaction between the polymers, morphology of the blends, crystallization rate and extent of crystallization. The mechanical properties of the final product of the blend depend on the crystal structure, morphology and melt history. The time required for the crystallization depends on the nucleation, cooling rate and temperature of melt processing. In order to control the rate of crystallization and the degree of crystallinity and to obtain the desired morphology and properties, efforts have been made to study the crystallization kinetics and determine change in material properties of various polymers [4-9]. Differential scanning calorimeter has been useful in studying the polymer crystallization kinetics [10-11].

A review by Gerard et al. [12] deals with the DSC method of analyzing the ability of PET to crystallize. An important conclusion obtained from this work is that the phase changes taking place in PET depend mainly on the thermal history of the sample. This fact has also been supported by Wasiak et al. [13]. This paper claims that temperature has an effect on the amount of crystals formed. Although a knowledge of the crystallization kinetics of polymeric materials is important from the aspects of both fundamental and practical applications, only few studies have been carried out on the crystallization behaviour and rheological characterization of PET/PMMA blends.

For studying the isothermal crystallinity a number of mathematical models [14-18] have been proposed. Unlike Avrami model, use of the Tobin [18] and Urbanovici-Segal [19] model to analyze the isothermal crystallization data of semi-crystallineamorphous polymers is rarely reported in the literature. Literature reports no previous work on the isothermal crystallization kinetic analysis of different compositions of PET/PMMA blends. Therefore, in the present work, all three macro kinetic models are used to analyze the isothermal crystal- lization data of three different compositions of PET/PMMA blends. The experimental data are fitted to each respective model using a nonlinear and a linear program to obtain the isothermal crystallization parameters. The goodness of the fit (measured using correlation coefficient, $r^{2}$ ) suggests the applicability of the model in describing the isothermal crystallization data of PET/PMMA blends.

\section{Experimental}

\subsection{Materials}

PET bottle grade was supplied in pellet form by Century Enka Pvt. Ltd. Pune, India. The intrinsic viscosity of the resin was 0.60 measured at $25^{\circ} \mathrm{C}$ in a 60/40 (V/V) phenol/tetrachloroethane mixture using a Cannon-Ubbelohde viscometer. Virgin PMMA injection molded in sheet form at an injection pressure of $1.03 \cdot 10^{8} \mathrm{~Pa}$, cylinder temperature, 177 to $210^{\circ} \mathrm{C}$ and mould temperature of $80^{\circ} \mathrm{C}$ was used.

\subsection{Sample Preparation}

PET and PMMA were dried for five hours at $100^{\circ} \mathrm{C}$ to ensure low moisture levels. PET/PMMA blends were prepared by the melt mixing method in a $500 \mathrm{ml}$ step polymerization reactor. Three different compositions of dried PET and PMMA (PET/ PMMA, 50/50, 75/25 and 90/10, weight/weight percent) were made by weighing the accurate quantities. The mixture was melt mixed in the reactor, in a nitrogen atmosphere of $20 \mathrm{ml} / \mathrm{min}$ for one hour at $275^{\circ} \mathrm{C}$. The stirrer speed was 100 r.p.m. The blended product was removed from the reactor and ground with a Moulinex super blender grater 3 at 400 r.p.m.

\subsection{Isothermal crystallization studies}

A Mettler-Toledo TA 4000 differential scanning calorimeter (DSC) was used to record the isothermal melt-crystallization exotherms as well as the subsequent melting endotherms for PET, PMMA and their blends. Each sample was used only once and all the runs were carried out in nitrogen atmosphere $(20 \mathrm{ml} /$ minute). Calibration of the temperature scale was done with pure indium standard $\left(T_{m}^{0}=156.6^{\circ} \mathrm{C}\right.$ and $\left.\Delta H_{f}^{0}=28.5 \mathrm{~J} \cdot \mathrm{g}^{-1}\right)$ on every sample to ensure accuracy and reliability of the data obtained. Temperature calibration of the DSC 
instrument was carried out with a standard pan containing indium, lead and zinc whose melting temperature are, $156.6, \quad 327.4$ and $419.5^{\circ} \mathrm{C}$ respectively. Samples $(10 \mathrm{mg})$ were dried completely at $100^{\circ} \mathrm{C}$ for five hours and then used for the analysis.

In a typical isothermal run, the sample was heated in DSC furnace from $30^{\circ} \mathrm{C}$ to $280^{\circ} \mathrm{C}$ at a heating rate of $10^{\circ} \mathrm{C} / \mathrm{min}$, under a nitrogen atmosphere. It was annealed for 10 minutes at $280^{\circ} \mathrm{C}$. The sample was then cooled to the predetermined isothermal temperature $\left(T_{c}\left[{ }^{\circ} \mathrm{C}\right]\right)$ at a cooling rate of $100^{\circ} \mathrm{C} / \mathrm{min}$. The sample was kept at the isothermal temperature till the baseline was attained. Kinetic analysis software available in the Mettler instrument was used to convert the data into Excel files which was later analyzed using, Avrami, Tobin and Urbanovici-Segal models for estimation of the isothermal kinetic parameters of the PET/PMMA blends.

\section{Theory}

Estimation of isothermal crystallization parameters is normally carried out using the data obtained from crystallization exotherms with a basic assumption that the amount of crystallinity obtained is linearly proportional to the heat released during crystallization. By integrating the heat evolved during crystallization, a relation between relative crystallinity and time can be obtained (Equation (1)).

$\theta(t)=\frac{\int_{0}^{t} \frac{\mathrm{d} H_{c}}{\mathrm{~d} t} \mathrm{~d} t}{\Delta H_{c}}$

were $t$ is the elapsed time and $\mathrm{d} H_{c}$ is the released heat during crystallization for small time interval $\mathrm{d} t$ and $\Delta H_{c}$ is the total enthalpy of crystallization for a specific crystallization temperature. $\Delta H_{c}$ can be given as Equation (2):

$$
\Delta H_{c}=\int_{0}^{\infty} \frac{\mathrm{d} H_{c}}{\mathrm{~d} t} \mathrm{~d} t
$$

The Avrami equation [14] given below (3) is mostly used to determine the time dependent relative crystallinity $\theta(t)$.

$$
\theta(t)=1-\exp \left(-k_{a} t^{n_{a}}\right)
$$

In Equation (3), $k_{a}$ is the Avrami rate constant and $n_{a}$ the Avrami exponent. $k_{a}$ and $n_{a}$ are temperature dependent parameters and are specific to a given crystalline morphology and type of nucleation [14]. The Tobin equation [16] is proposed on the basis of kinetic phase transformation and is given as Equation (4):

$\theta(t)=\frac{k_{t} t^{n_{t}}}{1+k_{t} t^{n_{t}}}$

$k_{t}$ and $n_{t}$ are the Tobin rate constant and Tobin exponent respectively. The Tobin exponent, $n_{t}$, is governed by different types of nucleation and growth mechanisms [16].

Urbanovici and Segal [19] developed a new kinetic equation, which is a modification of the Avrami model. The relationship between the time-dependent relative crystallinity function $\theta(t)$ and the crystallization time given by Urbanovici-Segal model is as shown in Equation (5):

$\theta(t)=1-\left[1+(r-1) k_{u s} t^{n_{u s}}\right]^{\frac{1}{1-r}}$

In this equation, $k_{u s}$ and $n_{u s}$ are the UrbanoviciSegal crystallization rate constant and exponent, respectively. $k_{u s}$ has the unit of (time) $)^{-1} . r$ is an optimization parameter which determines the extent of deviation for the Urbanovici-Segal equation from the Avrami equation. When $r$ approaches 1, the Urbanovici-Segal equation becomes similar to the Avrami equation [20].

Kinetic analysis of virgin PET, and PET/PMMA blends were carried out using Equations (3), (4) and (5).

The objective was to obtain the isothermal crystallization rate constant and the crystallization exponent respectively. Two different analysis methods were used. One of the procedures used Solver, a nonlinear optimization function found in Excel. In the other approach Equations (3) and (4) were linearized and applied to the experimental data to obtain the isothermal kinetic parameters.

The goodness of the methods were confirmed using correlation coefficient $\left(r^{2}\right)$. The greater the convergence of $r^{2}$ towards 1 the better is the quality of the fit.

The linearized logarithmic form of Equations (3) and (4) is represented as Equations (6) and (7):

$\ln [-\ln (1-\theta(t))]=n \ln (t)+\ln k$ 
$\ln \left(\frac{-\theta}{\theta-1}\right)=\ln k+n \ln t$

Plotting the first term in Equation (6) versus $\log t$ one can obtain $k$ and $n$ from the slope and intercept respectively. Similarly on plotting the first term in Equation (7) as a function of $\ln t$ the kinetic constants $k$ and $n$ can be determined.

\subsection{Isothermal crystallization kinetics of virgin PET and PET/PMMA blends}

Typical isothermal melt crystallization exotherms for PET90/PMMA10 [wt/wt\%] after isothermal crystallization at crystallization temperatures ranging from 207 to $221^{\circ} \mathrm{C}$ are shown in Figure 1. Other blends also showed similar crystallization behaviours. After thermal stabilization, the heat flow signal, on crystallization exhibited an exotherm, a result of the thermal energy released due to crystallization. The onset and endset of crystallization is determined by drawing a tangent to the respective baselines.

Figures 2 and 3 are typical figures illustrating the sigmoidal behaviour of virgin PET and the blend of PET90/PMMA10 [wt/wt\%]. As seen in Figures 2 and 3 the time needed to reach $100 \%$ relative crystallinity increased with increasing crystallization temperature, $T_{c} \cdot t_{0.5}$ is an important parameter which can be obtained from the plot of relative crystallinity and time. $t_{0.5}$ is defined as the time needed to attain $50 \%$ crystallinity. Table 1 summarizes the values of crystallization half-time $\left(t_{0.5}\right)$ using the Equation (8).

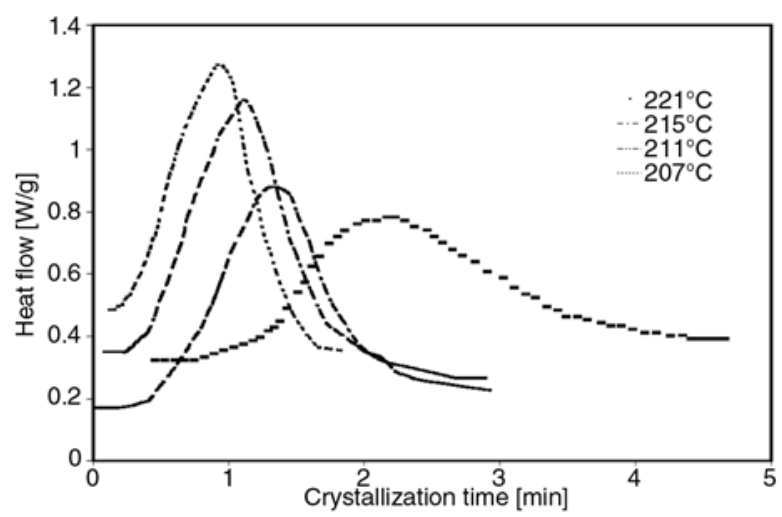

Figure 1. Isothermal melt crystallization exotherms for PET 90/PMMA10 observed at different crystallization temperatures $t_{0.5}=\left(\frac{\ln 2}{k}\right)^{\frac{1}{n}}$

The $t_{0.5}$ values shown in Table 1 are found to decrease with decreasing crystallization temperature, $T_{c}$. Figure 4 indicates a plot of $t_{0.5}$ versus crystallization temperature, $T_{c}$. The $t_{0.5}$ values are found to decrease as the PET composition decreases in the blends. PET virgin has the maximum $t_{0.5}$ value.

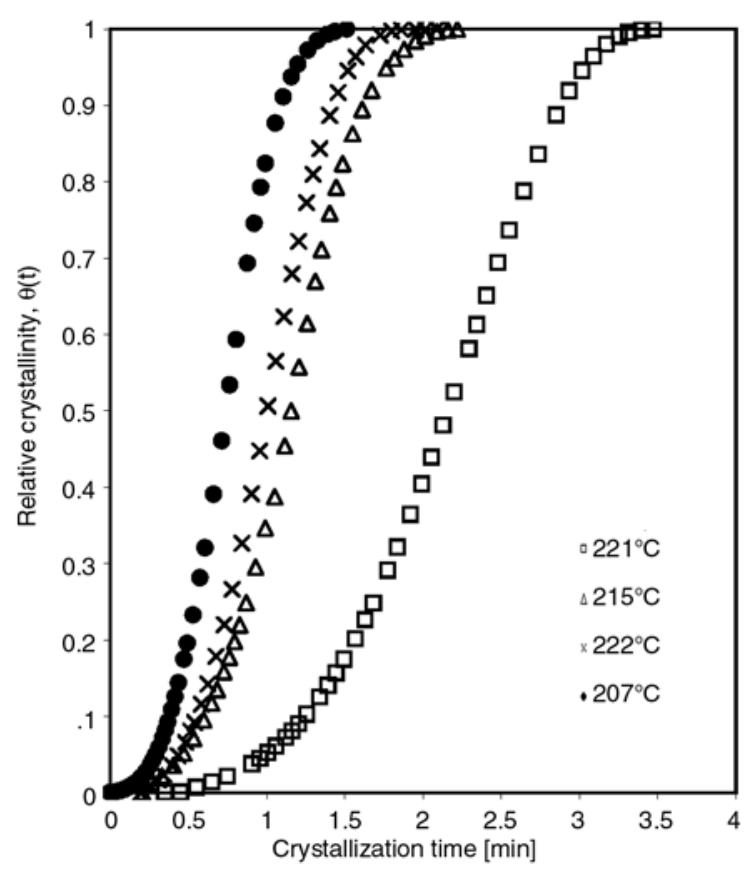

Figure 2. Relative crystallinity as a function of time of virgin PET

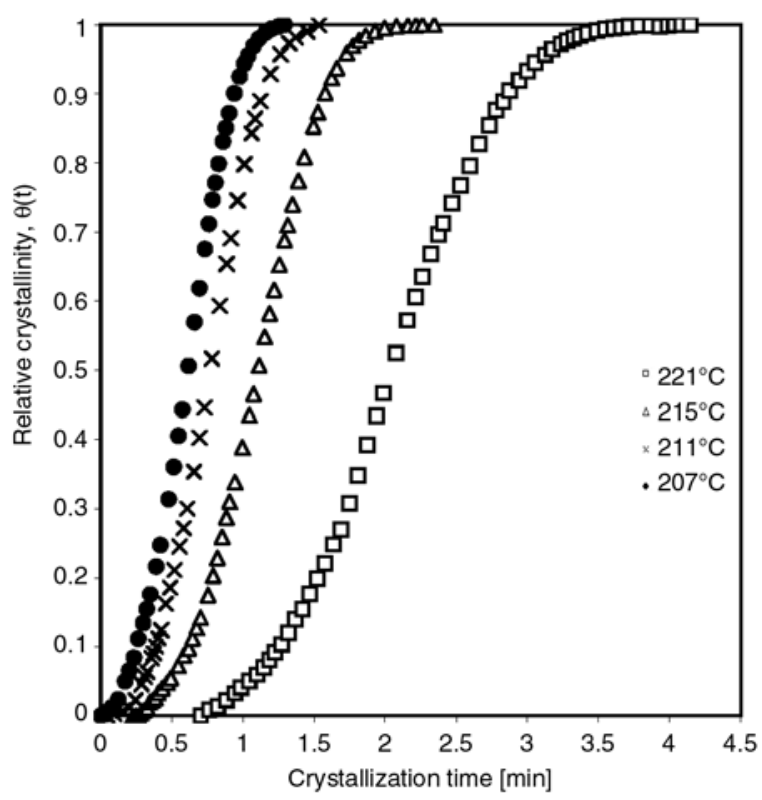

Figure 3. Relative crystallinity as a function of time of PET 90/PMMA10 [wt/wt\%] 
Table 1. Summary of isothermal crystallization parameters for virgin PET and its blends using Avrami model

\begin{tabular}{|c|c|c|c|c|c|c|c|c|c|c|c|}
\hline & \multirow[b]{2}{*}{$\begin{array}{c}\boldsymbol{T}_{\boldsymbol{c}} \\
{\left[{ }^{\circ} \mathbf{C}\right]}\end{array}$} & \multicolumn{5}{|c|}{$\begin{array}{l}\text { Isothermal kinetic constants } \\
\text { based on Solver method }\end{array}$} & \multicolumn{5}{|c|}{$\begin{array}{l}\text { Isothermal kinetic constants } \\
\text { based on linearization method }\end{array}$} \\
\hline & & $\begin{array}{c}k_{a} \\
{\left[\mathrm{~min}^{-1}\right]}\end{array}$ & $n_{a}$ & $\begin{array}{c}\text { Average } \\
n_{a}\end{array}$ & $r^{2}$ & $\begin{array}{c}t_{0.5} \\
{[\mathrm{~min}]}\end{array}$ & $\begin{array}{c}k_{a} \\
{\left[\mathrm{~min}^{-1}\right]}\end{array}$ & $n_{a}$ & $\begin{array}{c}\text { Average } \\
n_{a}\end{array}$ & $r^{2}$ & $\begin{array}{c}t_{0.5} \\
{[\mathrm{~min}]}\end{array}$ \\
\hline \multirow{4}{*}{ VIRGIN PET } & 221 & 0.04 & 3.78 & \multirow{4}{*}{3.33} & 1.00 & 2.14 & 0.04 & 3.78 & \multirow{4}{*}{3.33} & 1.00 & 2.14 \\
\hline & 215 & 0.45 & 3.30 & & 1.00 & 1.14 & 0.45 & 3.30 & & 1.00 & 1.14 \\
\hline & 211 & 0.70 & 3.30 & & 1.00 & 1.00 & 0.70 & 3.30 & & 1.00 & 1.00 \\
\hline & 207 & 1.74 & 2.97 & & 1.00 & 0.73 & 1.74 & 2.97 & & 1.00 & 0.73 \\
\hline \multirow{4}{*}{ PET90/PMMA10 } & 221 & 0.05 & 3.71 & \multirow{4}{*}{3.09} & 1.00 & 2.06 & 0.05 & 3.71 & \multirow{4}{*}{3.09} & 1.00 & 2.06 \\
\hline & 215 & 0.50 & 3.31 & & 1.00 & 1.10 & 0.50 & 3.31 & & 1.00 & 1.10 \\
\hline & 211 & 1.51 & 2.81 & & 1.00 & 0.76 & 1.51 & 2.81 & & 1.00 & 0.76 \\
\hline & 207 & 2.55 & 2.55 & & 1.00 & 0.60 & 2.55 & 2.55 & & 1.00 & 0.60 \\
\hline \multirow{4}{*}{ PET75/PMMA25 } & 221 & 0.44 & 4.36 & \multirow{4}{*}{3.80} & 1.00 & 1.11 & 0.44 & 4.35 & \multirow{4}{*}{3.80} & 1.00 & 1.11 \\
\hline & 215 & 6.67 & 4.31 & & 1.00 & 0.59 & 6.67 & 4.30 & & 1.00 & 0.59 \\
\hline & 211 & 8.81 & 3.58 & & 1.00 & 0.49 & 8.81 & 3.58 & & 1.00 & 0.49 \\
\hline & 207 & 12.43 & 2.96 & & 1.00 & 0.38 & 9.51 & 2.96 & & 1.00 & 0.41 \\
\hline \multirow{4}{*}{ PET50/PMMA50 } & 221 & 5.35 & 4.28 & \multirow{4}{*}{3.71} & 1.00 & 0.62 & 3.42 & 3.76 & \multirow{4}{*}{3.60} & 0.91 & 0.65 \\
\hline & 215 & 11.99 & 3.79 & & 1.00 & 0.47 & 11.97 & 3.79 & & 1.00 & 0.47 \\
\hline & 211 & 22.77 & 3.73 & & 1.00 & 0.39 & 22.77 & 3.73 & & 1.00 & 0.39 \\
\hline & 207 & 21.68 & 3.04 & & 1.00 & 0.32 & 21.68 & 3.04 & & 1.00 & 0.32 \\
\hline
\end{tabular}

$T_{c}$ - crystallization temperature $\left[{ }^{\circ} \mathrm{C}\right]$

According to Figure 4 it is obvious that for virgin PET and the polymer blends the crystallization half time increases with crystallization temperature. The analysis of half time of crystallization demonstrates that increasing concentration of PMMA in the blends lead to some kind of deceleration of the PET crystallization. This behaviour could be possibly caused by the decreasing segmental mobility of the PET chains in the presence of increasing amounts of PMMA.

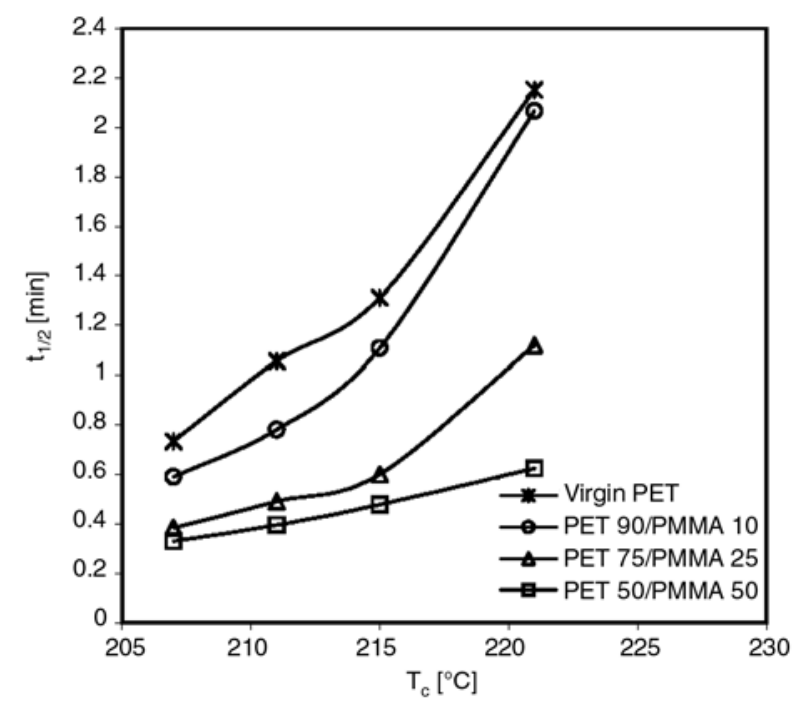

Figure 4. $t_{1 / 2}$ as a function of isothermal crystallization temperature for virgin PET and its blends

\subsection{Application of the Solver method to determine the isothermal crystallization parameters for PET/PMMA blends using the Avrami and Tobin models}

The Avrami Equation (3) was applied to the experimental data of virgin PET and PET/PMMA blends. Solver, a non-linear optimization method was used to obtain the isothermal crystallization parameters, $k_{a}$ and $n_{a}$ shown in Table 1. Figure 5 is a typical plot of relative crystallinity as a function of time for PET75/PMMA25 [wt/wt\%] blend fitted using the Avrami model. The Avrami model is found to fit the data well at 207,211 and $215^{\circ} \mathrm{C}$

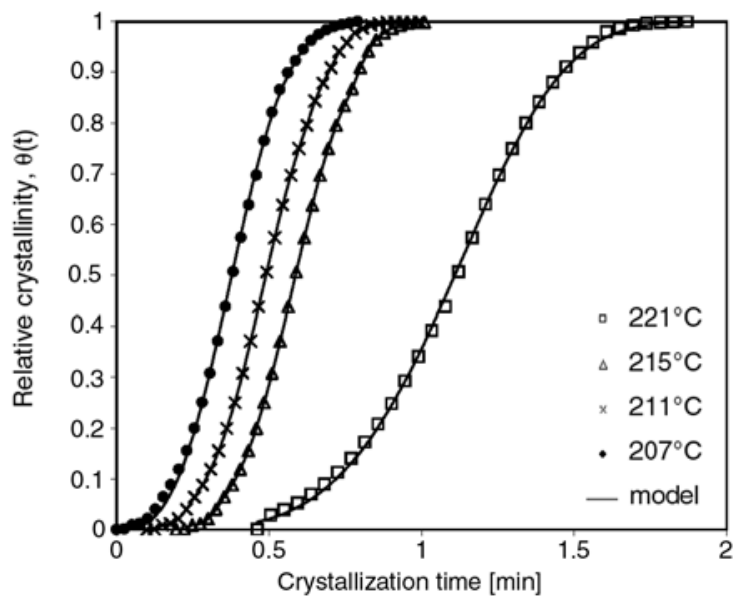

Figure 5. Plot of relative crystallinity as a function of time for PET 75/PMMA25 [wt/wt\%] using Avrami model 
while at $221^{\circ} \mathrm{C}$ the fitting is found to deviate slightly at the beginning and later stages of the conversion. The average value of the Avrami exponent for virgin PET and PET/PMMA blends is found to range from 3.09 to 3.80. $n_{a}$ is found to be temperature dependent and is found to increase with increasing, $T_{c}$.

The isothermal rate constant values in Table 1 exhibits sensitivity to change with crystallization temperature. It increases with decreasing crystallization temperature. This could plausibly be due to the fact that the concentration of the crystallizable material (PET) decreases in the blend. The $k_{a}$ values for PET50/PMMA50 are the highest compared to the other blends. This trend for PET50/PMMA50 could not be explained. A qualitative observation of Table 1 reveals that the $k_{a}$ values vary from 0.04 to $22.77 \mathrm{~min}^{-1}$.

The Tobin model (Equation (4)) was applied to the experimental data to obtain the Tobin's isothermal kinetic parameters, $k_{t}$ and $n_{t}$. Figure 6 is a typical figure indicating the application of Tobin model using the Solver method. Table 2 summarizes the Tobin kinetic parameters along with the correlation coefficient $\left(r^{2}\right)$ values obtained as a result of the best fit. According to Table 2 the Tobin exponent is found to range from 3.88 to 6.56 . The Tobin exponents are found to be greater than Avrami exponents. The $r^{2}$ values obtained using Tobin equation are lower than the Avrami equation which indicates

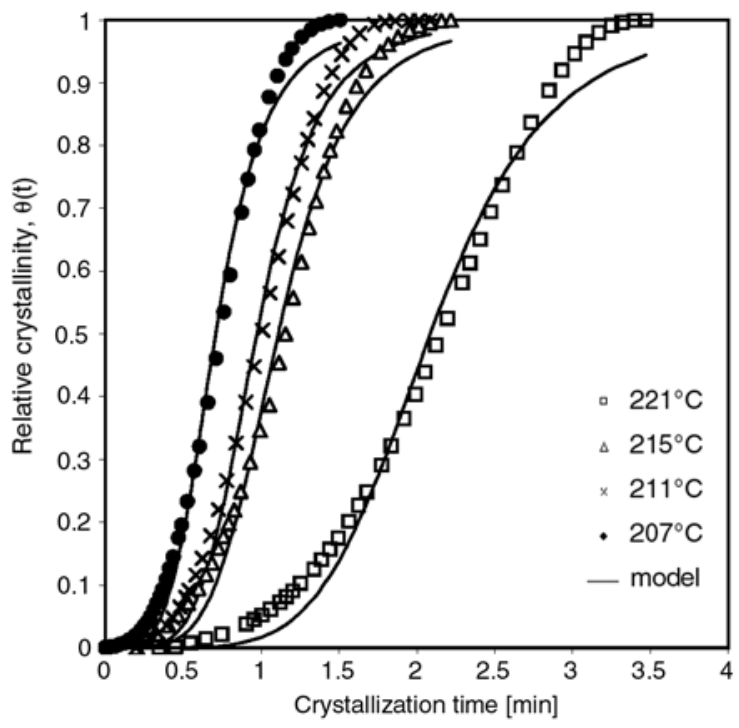

Figure 6. Plot of relative crystallinity as a function of time for virgin PET

that Tobin model has a poor fit compared to Avrami model.

Figure 7 is a typical plot showing the application of Equation (6) to PET75/PMMA25 data. The $r^{2}$ values and the kinetic constants obtained on fitting Equation (6) to the experimental data are given in Table 1 . The $r^{2}$ values are 1 indicating that the fit is $100 \%$ perfect. The crystallization exponent is found to decrease with decreasing temperature. The average exponent values are found to lie in the range of 3.09 to 3.80 . The isothermal rate constants are found to increase with decreasing crystallization

Table 2. Summary of isothermal crystallization parameters for virgin PET and its blends using Tobin model

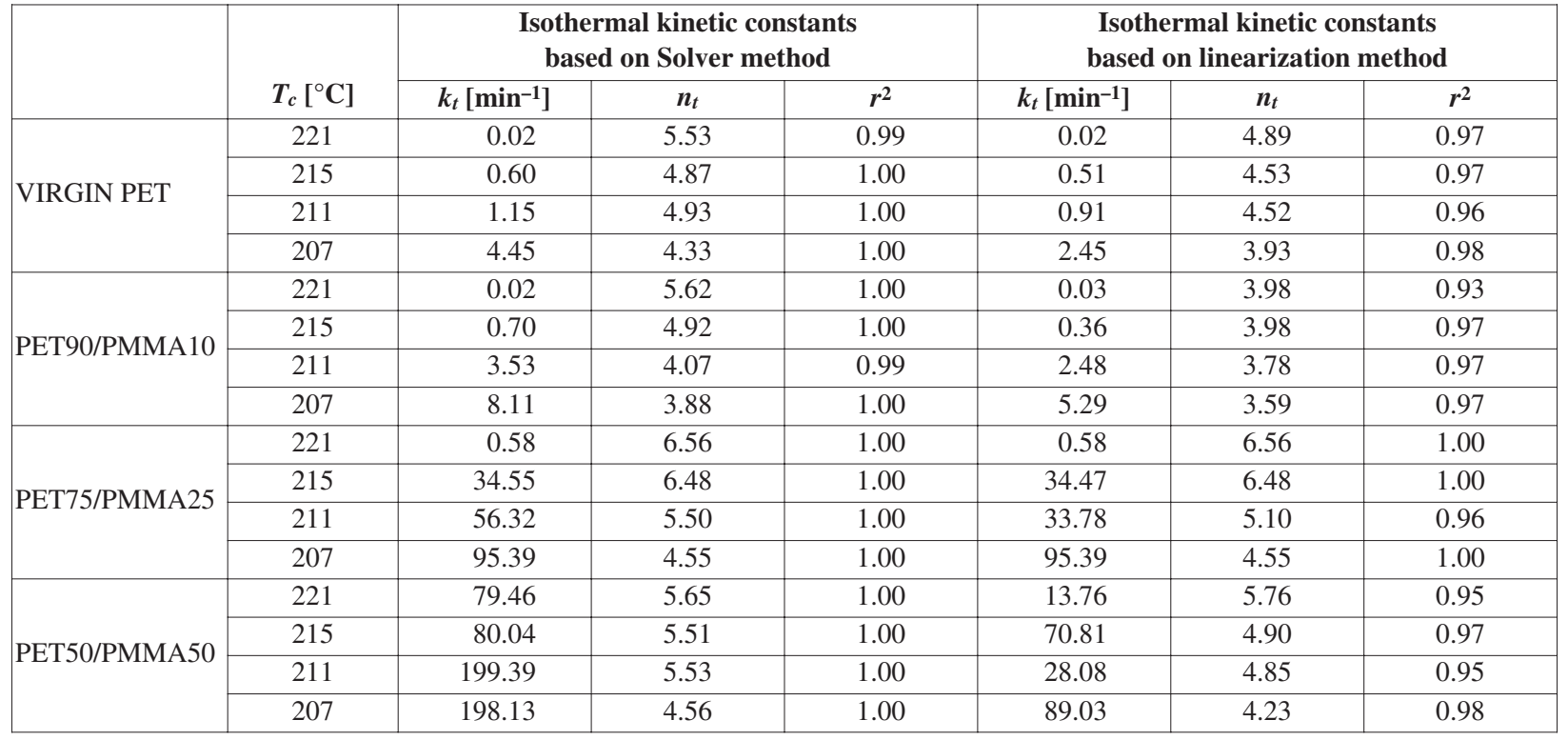

$T_{c}$ - crystallization temperature $\left[{ }^{\circ} \mathrm{C}\right]$ 


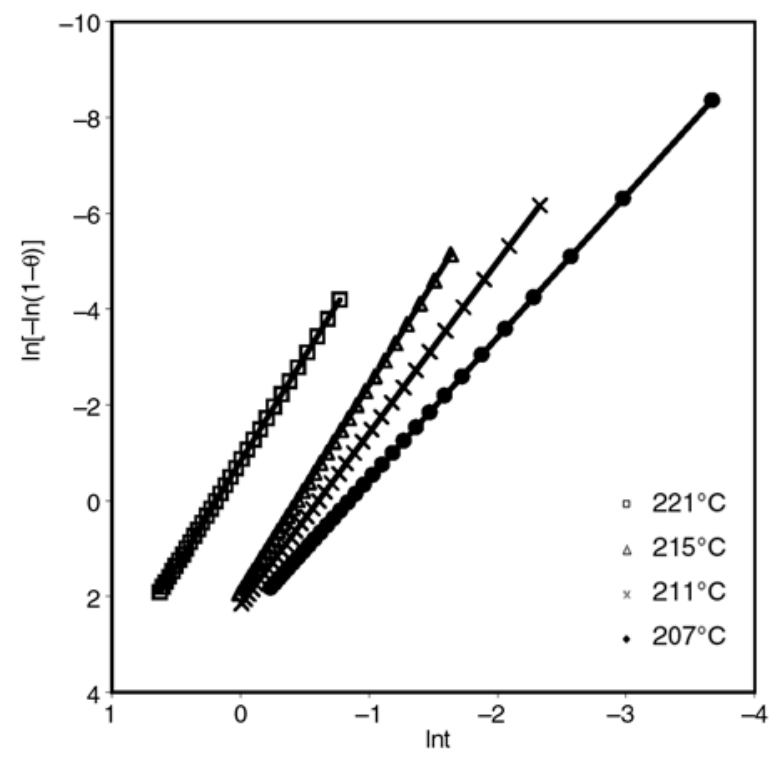

Figure 7. Plot of $\ln [-\ln (1-\theta)]$ as a function of logarithmic time for PET 75/PMMA25 [wt/wt\%] using Avrami Model

temperatures. The highest value of rate constant is found to be for virgin PET at $211^{\circ} \mathrm{C}$ and is approximately $22.77 \mathrm{~min}^{-1}$.

Figure 8 is a typical plot showing the application of Equation (7) to virgin PET. The correlation coefficient values and the kinetic constants obtained on fitting Equation (7) to the experimental data is given in Table 2. The isothermal exponent for crystallization is found to range between 3.59 to 6.60 . The highest exponent values are found for PET75/ PMMA25. The isothermal rate constants are found to increase with decreasing crystallization temperatures. The rate constant values are found to lie

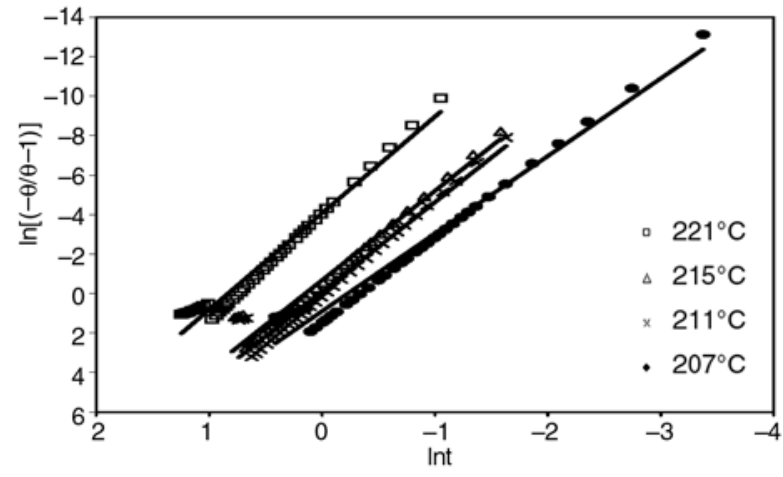

Figure 8. Plot of $\ln [-\theta /(\theta-1)]$ as a function of logarithmic time for PET virgin using Tobin model

between 0.02 to $95.39 \mathrm{~min}^{-1}$. Since the values of $r^{2}$ for the Tobin model lies between 0.93 to 1 the kinetic parameters obtained using this method is questionable.

\subsection{Isothermal crystallization kinetics of virgin PET and PET/PMMA blends based on the Urbanovici-Segal analysis}

The analysis based on Urbanovici-Segal model is done by fitting Equation (5) to the $\theta(t)$ obtained for each crystallization temperature. The UrbanoviciSegal kinetic parameters (i. e., $n_{u s}, k_{u s}$ and $r_{u s}$ ) and the $r^{2}$ values are summarized in Table 3. The $r^{2}$ values for the fit are found to be approximately 1.00 . Figure 9 is a typical plot of relative crystallinity as a function of time for virgin PET based on Urbanovici-Segal model. The average value of the exponent $\left(n_{u s}\right)$ for isothermal crystallization is

Table 3. Summary of isothermal crystallization parameters for virgin PET and its blends using Urbanovici-Segal model

\begin{tabular}{|c|c|c|c|c|c|c|c|}
\hline & $\boldsymbol{T}_{c}\left[{ }^{\circ} \mathbf{C}\right]$ & $r_{u s}$ & $k_{u s}\left[\min ^{-1}\right]$ & $n_{u s}$ & Average $n_{u s}$ & $r^{2}$ & $t_{0.5}[\mathrm{~min}]$ \\
\hline \multirow{4}{*}{ VIRGIN PET } & 221 & 0.75 & 0.41 & 3.60 & \multirow{4}{*}{3.30} & 1.00 & 2.15 \\
\hline & 215 & 0.80 & 0.67 & 3.35 & & 1.00 & 1.31 \\
\hline & 211 & 0.86 & 0.83 & 3.16 & & 1.00 & 1.06 \\
\hline & 207 & 1.01 & 1.21 & 3.05 & & 1.00 & 0.73 \\
\hline \multirow{4}{*}{ PET90/PMMA10 } & 221 & 0.91 & 0.43 & 3.38 & \multirow{4}{*}{2.76} & 1.00 & 2.07 \\
\hline & 215 & 0.75 & 0.77 & 2.85 & & 1.00 & 1.11 \\
\hline & 211 & 0.70 & 1.06 & 2.47 & & 1.00 & 0.78 \\
\hline & 207 & 0.91 & 1.43 & 2.34 & & 1.00 & 0.59 \\
\hline \multirow{4}{*}{ PET75/PMMA25 } & 221 & 0.81 & 0.80 & 3.92 & \multirow{4}{*}{3.52} & 1.00 & 1.12 \\
\hline & 215 & 0.89 & 1.50 & 3.85 & & 1.00 & 0.60 \\
\hline & 211 & 1.10 & 1.87 & 3.74 & & 1.00 & 0.49 \\
\hline & 207 & 0.76 & 2.19 & 2.58 & & 1.00 & 0.38 \\
\hline \multirow{4}{*}{ PET50/PMMA50 } & 221 & 0.78 & 1.43 & 3.87 & \multirow{4}{*}{3.25} & 1.00 & 0.62 \\
\hline & 215 & 0.67 & 1.80 & 3.20 & & 1.00 & 0.48 \\
\hline & 211 & 0.73 & 2.20 & 3.30 & & 1.00 & 0.40 \\
\hline & 207 & 0.68 & 2.53 & 2.65 & & 1.00 & 0.33 \\
\hline
\end{tabular}

$T_{c}$ - crystallization temperature $\left[{ }^{\circ} \mathrm{C}\right]$ 


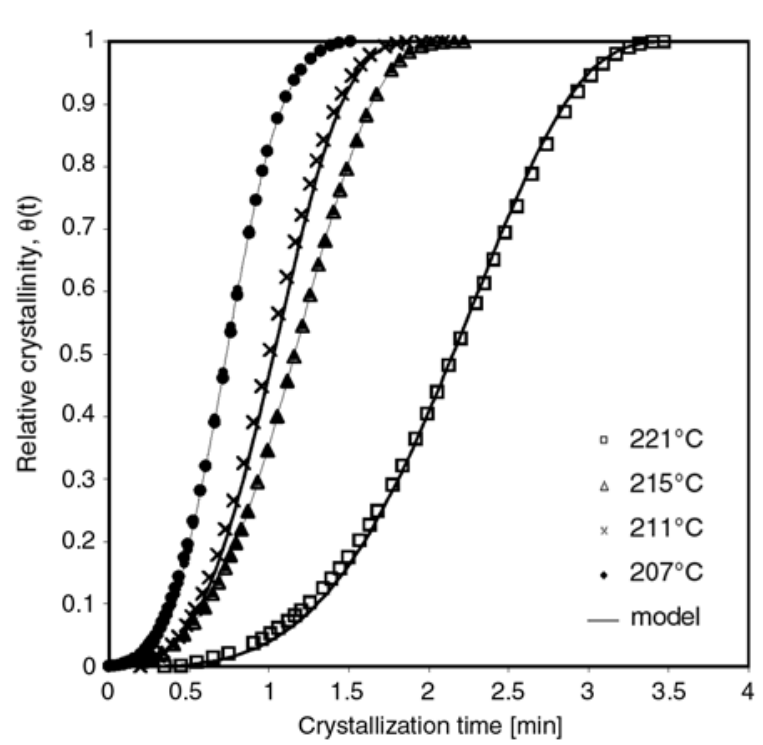

Figure 9. Relative crystallinity as a function of time for virgin PET using Urbanovici-Segal model

found to vary from 2.76 to 3.52 . This may correspond to spherulitic growth [20].

The Urbanovici-Segal rate constant, $k_{u s}$ increases with decreasing crystallization temperature. This trend is similar to that indicated by Avrami and Tobin models.

$r$ in Urbanovici-Segal model is the parameter which fine tunes the fit of the model to the experimental data. This could be the reason why the Urbanovici-Segal model provides a better fit to the data. $k_{a}$ values (Table 1) are generally found to be higher than $k_{u s}$ values (Table 3) especially for PET/PMMA blends.

The $n_{a}$ and $n_{u s}$ values obtained using the Avrami and Urbanovici-Segal model are in general found to be comparable. Based on the $r^{2}$ values and the physical observation of the plots Urbanovici-Segal model is found to be a good model for describing the isothermal crystallization kinetics of virgin PET and PET/PMMA blends.

The equation used to calculate the $t_{0.5}$ values for the Urbanovici-Segal model is given in Equation (9):

$t_{0.5}=\left(\frac{0.5^{(1-r)}-1}{r-1}\right) \frac{1}{k_{u s}}$

where $k_{u s}$ and $r$ have the same significance as indicated in Equation (5). The $t_{0.5}$ values have a similar trend to that of Avrami model.

The values obtained using Equation (9) are given in Table 3.

\subsection{Overall comparison between Avrami, Tobin and Urbanovici-Segal using the Solver and linearization method}

The efficacy of each kinetic equation in describing the experimental data is represented by the $r^{2}$ value. The closer the $r^{2}$ value to 1 the better is the quality of the fit. Physical observation of the plots and the $r^{2}$ values indicate that Urbanovici-Segal and Avrami method using Solver is a good multivariable regression program to fit the experimental data to the aforementioned models. Figure 6 and the $r^{2}$ values in Table 2 clearly show the deviation of the Tobin model from the experimental data. The Tobin model appears to mismatch the data for the complete experimental range. A typical example for the comparison between the three models is shown in Figure 10. If a comparison is made between Avrami and Urbanovici-Segal models a conclusion can be arrived that the Arvami model over-estimates the $\theta(t)$ in the middle stage (Figure 10) and underestimates it at the early and later stages of the crystallization.

No similarity exists between the isothermal kinetic constants estimated using the Solver and linearization method for the Avrami and the Tobin model. The Avrami exponent values are lower compared to the Tobin values. The conclusion which can be drawn based on the $r^{2}$ value is that Avrami and Urbanovici-Segal equation are good models to predict the isothermal kinetic constants for the

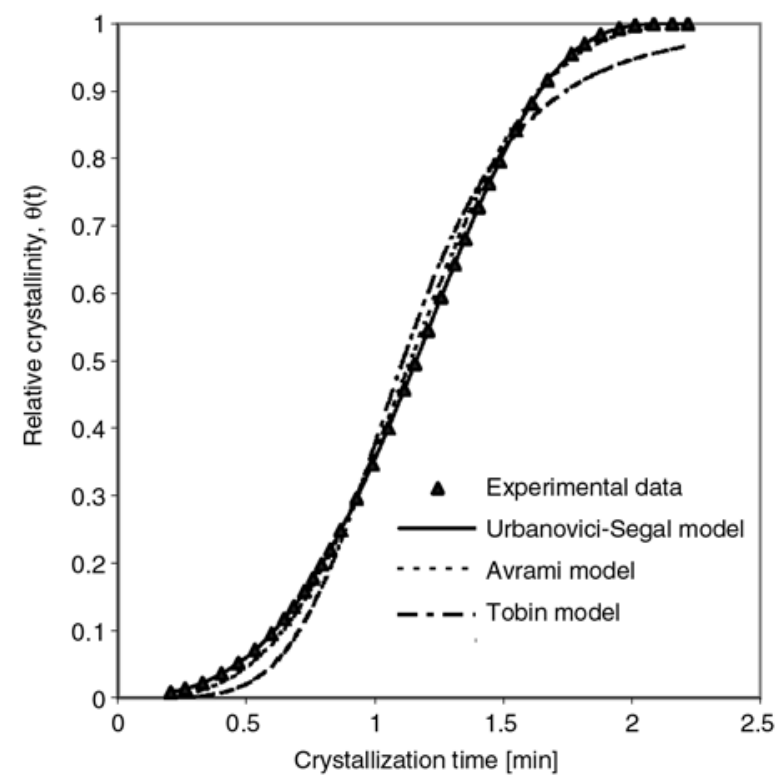

Figure 10. Modeling of virgin PET data using three different models at $215^{\circ} \mathrm{C}$ 
PET/PMMA systems using both the Solver and linearization methods.

Identical crystallization rate constant values are obtained using Avrami model when the Solver and linearization methods were applied to the data obtained for the blends and virgin PET. To further substantiate the observation the rate constant and exponent values obtained using Tobin model were plugged into the respective equations and replotted against the relative crystallinity data of PET90/

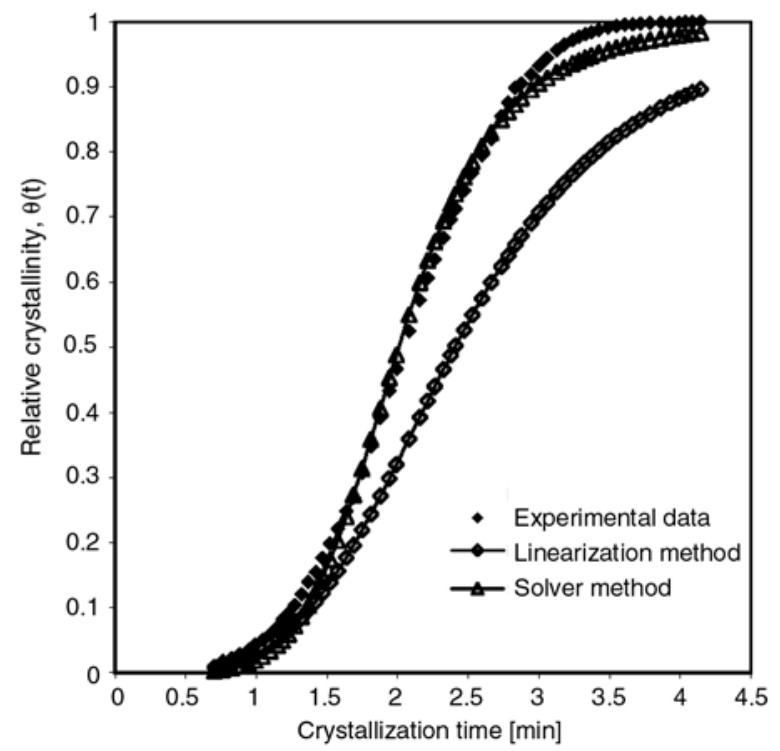

Figure 11. Comparison of the experimental data with Tobin model for PET 90/PMMA10 at $221^{\circ} \mathrm{C}$ using kinetic constants generated through Solver and linear fitting method

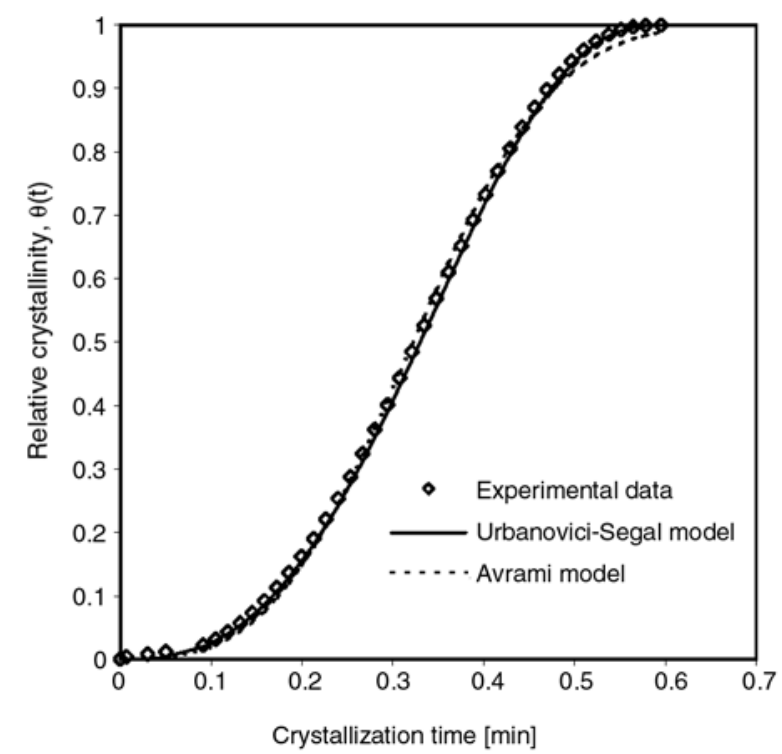

Figure 12. Comparison of the experimental data with Avrami and Urbanovici-Segal models for PET 50/PMMA50 at $207^{\circ} \mathrm{C}$ using kinetic constants generated through Solver
PMMA10. A typical plot depicting the compliance of the two different methods is indicated in Figure 11. As seen from the figure the Solver method seems to be more adaptable to determine the crystallization kinetic parameters of the system under investigation.

Figure 12 is a plot showing the compliance of Urbanovici-Segal model to a typical blend, (PET50/PMMA50), using the Solver method. The figure clearly indicates that the error between the experimental data and Urbanovici-Segal model is less compared to the Avrami model.

\subsection{Temperature-dependence of the kinetic parameters}

The Avrami parameter $n_{a}$ depends on the crystallization temperature and the shape of the crystals being grown. The Avrami parameter, $n_{a}$, of virgin PET and PET/PMMA blends show fractional values (Tables 1 and 3). Such types of values are theoretically accounted in the general statistical Avrami theory of crystallization [21]. The Avrami theory supposes a partial overlapping of primary nucleation and crystal growth. In the case of diffusion controlled growth or other constraints on crystallization, fractional values could be found [21]. As seen in Tables 1 and 3 the isothermal crystallization exponent increases slightly with increasing temperature, plausibly indicating that diffusion controlled process might not be controlling the crystallization

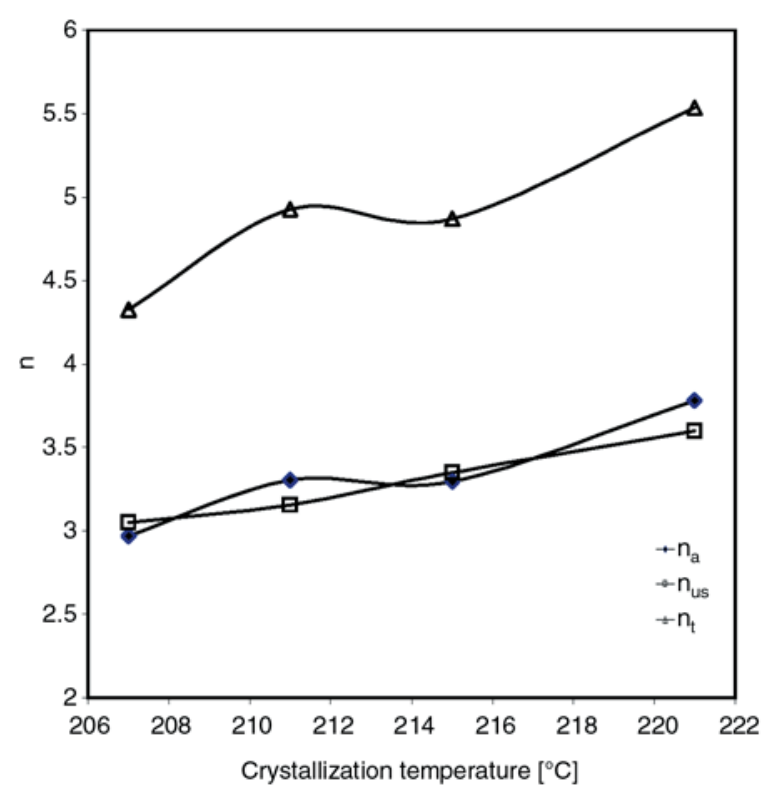

Figure 13. Crystallization exponent $(n)$ obtained for different models as a function of crystallization temperature for virgin PET 
mechanism in the present work [22]. Variation of $n_{a}, n_{t}$ and $n_{u s}$ as a function of crystallization temperature is shown in Figure 13.

Tables 1 and 3 lists the average isothermal crystallization exponents obtained for the virgin polymers and the blends. The average isothermal crystallization exponent for PET is found to be approximately 3.33 for an isothermal temperature range of 207$221^{\circ} \mathrm{C}$

Xanthos et al. [23] and Kim and Kim [24] reported an average value of PET to be around 2.35 and 2.37 respectively. They report that the Avrami parameter $n_{a}$ depends on the crystallization temperature and the shape of the crystals being grown. Literature cites different values of $n_{a}$ for different polymeric systems. Even for a similar system different values of $n_{a}$ have been reported. For example poly(3-hydroxy butyrate) (PHB) and its blends have an $n_{a}$ of approximately 2 as reported by Dubini et al. [25]. Mansour et al. [26] reported the value of $n$ close to 4. An et al. [27] observed the value of $n$ to be 4 for PHB and $n$ equal to 3 for $\mathrm{PHB} /$ polyvinyl alcohol blends.

When the temperature is low crystallization rate is controlled by diffusion of molecules at the crystal growth front (diffusion control); where as at temperatures closer to the melt temperature, as used in this study the rate limiting step of the crystallization process is the nucleation rate (nucleation control).

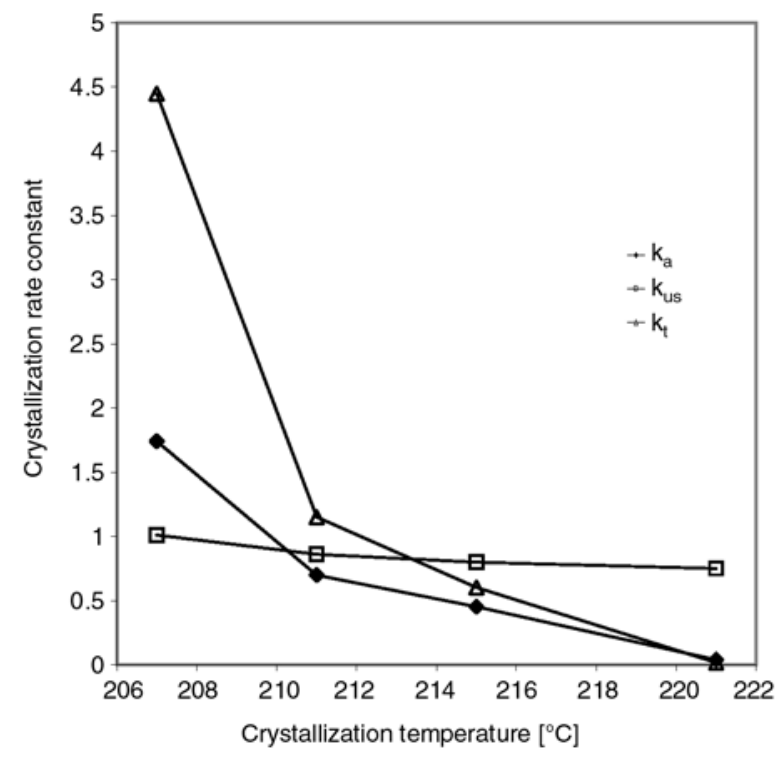

Figure 14. Crystallization rate constant obtained for different models as a function of crystallization temperature for virgin PET
Figure 14, illustrates the temperature-dependence of all the crystallization rate parameters (i. e., $k_{a}, k_{t}$ and $k_{u s}$ ). It is seen that the crystallization rate parameters exhibit temperature-dependence. It is worth noting that all the rate parameters have dimensions of $\mathrm{min}^{-1}$ and follow a defined pattern with increasing crystallization temperature.

\section{Conclusions}

The isothermal crystallization kinetics of virgin PET and PET/PMMA blends has been examined using DSC at crystallization temperatures ranging between 207 to $221^{\circ} \mathrm{C}$. Avrami, Tobin and Urbanovici-Segal models were used to determine the isothermal crystallization parameters for virgin PET and PET/PMMA blends using the Solver and linearization method. $r^{2}$ value was used to determine the goodness of the model. The crystallization exotherms followed the Avrami and the Urbanovici-Segal model with exponents ranging between 3.09 to 3.80 . The crystallization rate constants for the Avrami and Urbanovici-Segal model ranged between 0.04 to 22.77 and 0.41 to $2.53 \mathrm{~min}^{-1}$. The crystallization rate parameters $\left(k_{a}\right.$ and $k_{u s}$ ) determined on the basis of different macrokinetic models exhibit temperature dependence. Within the crystallization temperature range studied (i. e., 207 to $221^{\circ} \mathrm{C}$ ) the values of the rate parameters were all found to increase with decreasing temperature for the PET/PMMA blends and virgin PET.

\section{Acknowledgements}

The author would like to thank Dr. Johnson Mathew and Bader Masiar for their professional help during the course of this work. The author would also like to thank the Research Administration of Kuwait University for providing funds from project EC 02/05 for carrying out this work.

\section{References}

[1] Bishara A., Shaban H. I.: Nonisothermal crystallization kinetics of poly(ethylene terephthalate) and poly(methyl methacrylate) blends. Journal of Applied Polymer Science, 101, 3565-3571 (2006).

[2] Al-Mulla A., Shaban H.: Degradation kinetics of poly(ethylene terephthalate) and poly(methyl methacrylate) blends. Polymer Bulletin, 58, 893-902 (2007). 
[3] Dewangan B., Jagtap R. N.: Amphiphilic block copolymers of PtBA-b-PMMA as compatibilizers for blends of PET and PMMA. Polymer Engineering and Science, 46, 1147-1152 (2006).

[4] Jabarin S. A.: Crystallization kinetics of polyethylene terephthalate. II. Dynamic crystallization of PET. Journal of Applied Polymer Science, 34, 97-102 (1987).

[5] Gümther B., Zachmann H. G.: Influence of molar mass and catalysts on the kinetics of crystallization and on the orientation of poly(ethylene terephthalate). Polymer, 24, 1008-1014 (1983).

[6] Mehta A., Gaur U., Wunderlich B.: Equilibrium melting parameters of poly(ethylene terephthalate). Journal of Polymer Science Part B: Polymer Physics, 16, 289-296 (1978).

[7] Ozawa T.: Kinetics of non-isothermal crystallization. Polymer, 12, 150-158 (1971).

[8] Misra A., Stein R. S.: Light scattering studies of the early stages of the crystallization of poly(ethylene terephthalate). Journal of Polymer Science Part B: Polymer Letters, 10, 473-477 (1972).

[9] Stein R. S., Misra A.: Kinetics of growth of developing spherulites. Journal of Polymer Science Part B: Polymer Physics, 11, 109-116 (1973).

[10] Booth A., Hay J. N.: The use of differential scanning calorimetry to study polymer crystallization kinetics. Polymer, 10, 95-104 (1969).

[11] Hay J. N., Mills P. J.: The use of differential scanning calorimetry to study polymer crystallization kinetics. Polymer, 23, 1380-1384 (1982).

[12] Gerard D., Garda M-R., Saiter J-M.: Experimental protocol to determine the ability of PET to crystallize. Journal of Thermal Analysis, 7, 155-160 (2003).

[13] Wasiak A., Sajkiewicz P., Wozniak A.: Effect of cooling rate on crystallinity of i-propylene and polyethylene terephthalate crystallized in non-isothermal conditions. Journal of Polymer Science Part B: Polymer Physics, 37, 2821-2827 (1999).

[14] Avrami M.: Kinetics of Phase Change. I General Theory. Journal of Chemical Physics, 7, 1103-1112 (1939).

[15] Evans U. R.: The laws of expending circles and spheres in relation to the lateral growth of surface films and the grain-size of metals. Transactions of the Faraday Society, 41, 365-374 (1945).
[16] Tobin M. C.: Theory of phase transition kinetics with growth site impingement. I. Homogeneous nucleation. Journal of Polymer Science Part B: Polymer Physics, 12, 399-406 (1974).

[17] Malkin Y. A., Beghishev V. P., Keapin I. A., Bolgov S. A.: General treatment of polymer crystallization kinetics - Part 1. A new macrokinetic equation and its experimental verification. Polymer Engineering and Science, 24, 1396-1401 (1984).

[18] Tobin M. C.: Theory of phase transition kinetics with growth site impingement. III. Mixed heterogeneoushomogeneous nucleation and nonintegral exponents of the time. Journal of Polymer Science Part B: Polymer Physics, 15, 2269-2270 (1977).

[19] Urbanovici E., Segal E.: New formal relationships to describe the kinetics of crystallization. Thermochimica Acta, 171, 87-94 (1990).

[20] Supahol P., Simoaon P., Sirivat A.: Effect of crystalline and orientational memory phenomena on the isothermal bulk crystallization and subsequent melting behavior of poly(trimethylene terephthalate). Polymer International, 53, 1118-1126 (2004).

[21] Mandelkern L.: Crystallization of Polymers. McGrawHill, New York (1994).

[22] Arroyo M., Lopez-Manchado M. A. Avalos F.: Crystallization kinetics of polypropylene: II. Effect of the addition of short glass fibers. Polymer, 38, 5587-5593 (1997).

[23] Xanthos M., Baltzis B. C., Hsu P. P: Effects of carbonate salts on crystallization kinetics and properties of recycled poly(ethylene terephthalate). Journal of Applied Polymer Science, 64, 1423-1435 (1997).

[24] Kim S. P, Kim S. C.: Crystallization kinetics of poly(ethylene terephthalate): memory effect of shear history. Polymer Engineering and Science, 33, 83-91 (1993).

[25] Dubini E., Beltrame M., Conetti A., Saves B., Martuscelli E.: Crystallization and thermal behaviour of poly (D(-) 3-hydroxybutyrate)/poly(epichlorohydrin) blends. Polymer, 34, 996-1001 (1993).

[26] Mansour A. A., Saad G. R., Hamed A. M.: II. Dielectric investigation of cold crystallization of poly(3hydroxybutyrate) and poly(3-hydroxybutyrate-co-3hydroxyvalerate). Polymer, 40, 5377-5391 (1999).

[27] An Y., Li L., Dong L., Mo Z., Feng Z.: Nonisothermal crystallization and melting behavior of poly $(\beta$-hydroxybutyrate)-Poly(vinyl-acetate) blends. Journal of Polymer Science Part B: Polymer Physics, 37, 443-450 (1999). 